Please send trade news information and illustrations to Arveen Bajaj at the $B D J$, Nature Publishing Group, The Macmillan Building, 4-6 Crinan Street, London N1 9XW.

Trade news is provided as a service to readers using text and images from the manufacturer, supplier or distributor and does not imply endorsement by the $B D J$. Normal and prudent research should be exercised before purchase or use of any product mentioned.

\section{High intensity light}

GC has developed the brand new G-Light that uses the latest advances in LED technology. The G-Light's blue and violet LEDs give you two different wavelength peaks at the same time ensuring optimum curing of all of your VLC materials.

With a high intensity, narrow spectrum of light, the G-Light emits the maximum amount of light energy right to the tip of the handpiece. This re-chargeable light can be used over 400 times for ten seconds without any reduction in intensity and the constant light energy guarantees efficient and deep curing.

The lightweight, ergonomically designed, cordless curing light can be set to a range of practical curing times, including five, ten and 20-second intervals, as well as continuous mode to suit your individual requirements.

Reader response number 51

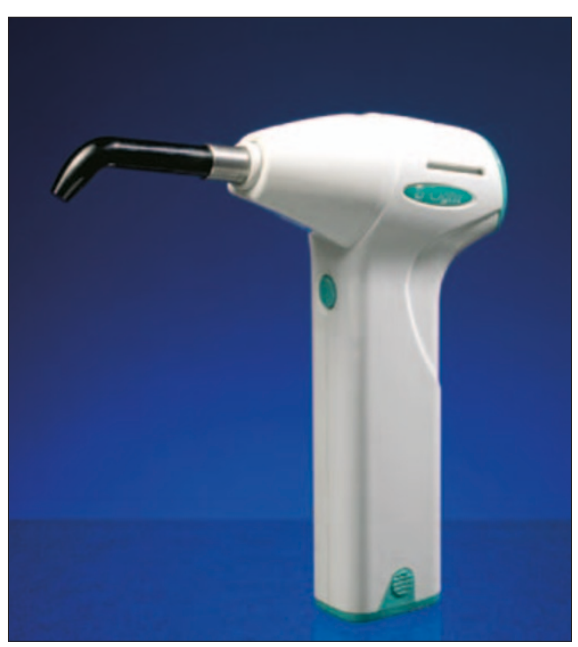

\section{Even retraction}

KerrHawe's new OptiView is an innovative lip and cheek retractor that has been designed to provide an even retraction of lips and cheeks, providing greater access to buccal and gingival areas simultaneously. Most cheek retractors achieve retraction that gives a limited 'oval' shape to the mouth whereas OptiView offers an even circular-style retraction of both lips and

\section{TRADE NEWS}

WHAT'S NEW

\section{Effervescent action}

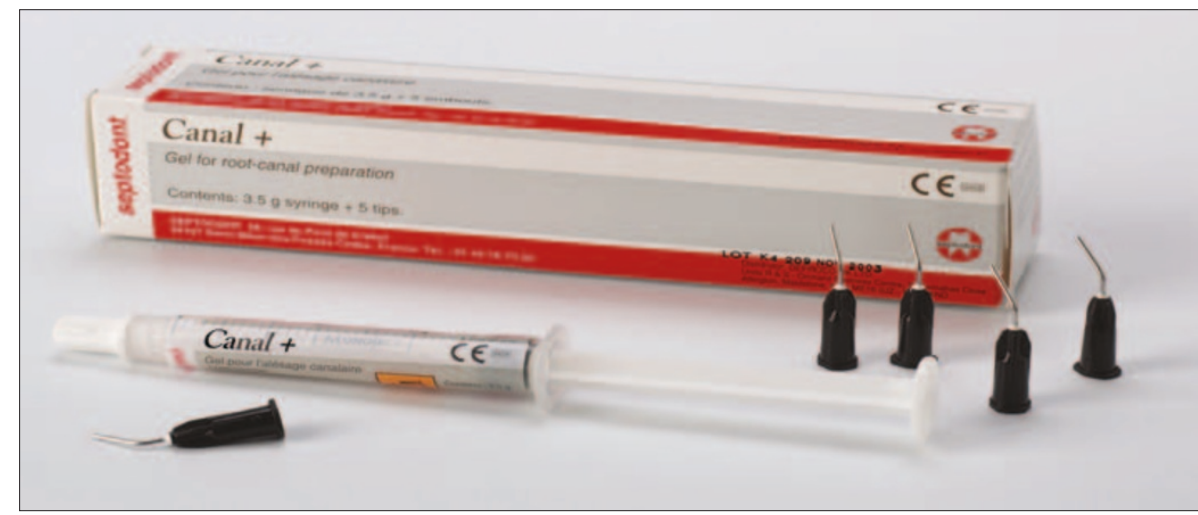

Canal+ from Septodont is a collating agent which can be used for root canal preparation. Its special properties ensure that the root canal is lubricated, making endodontic instrument penetration far easier; shaping is more efficient and cleaning of the root canal is easier as a result of the product's effervescent nature.

Because Canal+ has been specifically formulated for endodontic use, it offers you

and your patient the benefits of an EDTAbase for easy penetration into calcified areas, easy elimination by rinsing, simple application with a pre-filled syringe and disposable application tips for cross contamination control.

The product makes reaming easy making blunt endodontic instruments less likely.

Reader response number 50

\section{Fluid resistancy}

Sultan Chemists has recently launched its Com-Fit Groovy Mask. The product has a high level of fluid resistance to provide the maximum protection against blood, aerosols and other body fluids. It has improved breathability over leading fluid resistant masks and allows the wearer to be cool and comfortable. The mask has a 99.9\% particulate filtration at 0.1 micron and 99\% bacterial filtration at 3.0 micron to ensure a high level of protection against bacteria, viruses and other microscopic particles. The Groovy Mask comes in a color scheme of predominantly light blue, with purples and greens. Reader response number 52

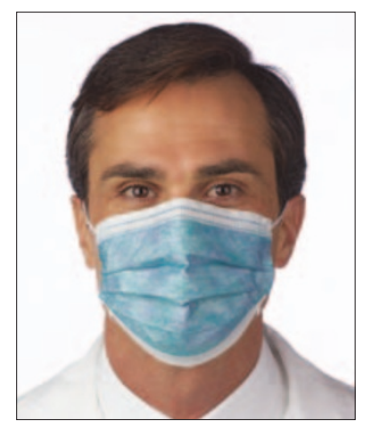

cheeks. The anatomical shaped lip holders with opening for the frenulum guarantee optimum comfort for the patient. The lateral flaps gently hold the cheeks and ease removal from the mouth.

The auxiliary aid allows the working field to be enlarged easily, effectively and comfortably and is also autoclavable.

Reader response number 53

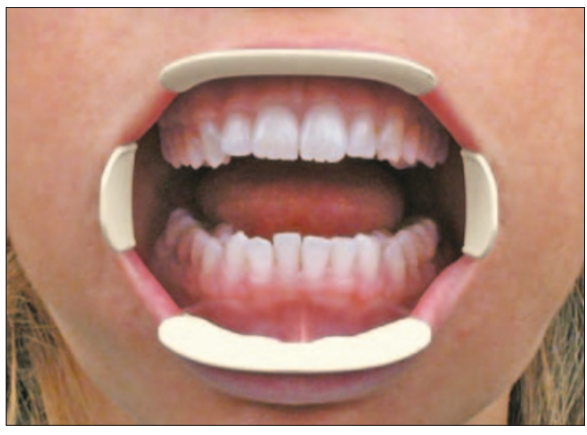




\section{Stability during processing}

Kodak Insight Dental Film is part of Kodak's range of Intraoral Dental Films. It has a radiation dose up to $60 \%$ lower than Kodak Ultra-Speed film, increased stability during processing which means fewer repeat $\mathrm{x}$-rays and improved image quality with high resolution and high contrast levels. They are available in several sizes for adults, adolescents and children and in a variety of packaging, including Super Poly-Soft packets, Bite-wing film packets and Two-film packets.

Reader response number 54

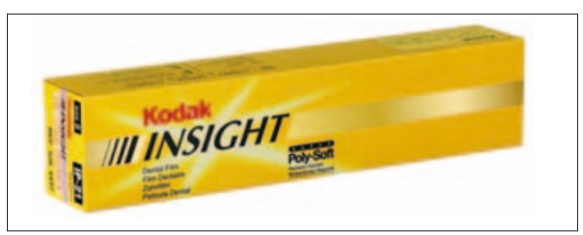

\section{Tailored solutions}

Independent specialists The Dental Imaging Company offer a range of products from leading manufacturers including Kodak Dental Systems, Dürr Dental, Satelec, Digora, Dexis and Medivision. Because it is independent, the company gives users the opportunity to compare the latest digital technology in order to make an informed decision over which product is right for them. It can also help you tailor a solution to meet the needs of your practice, rather than sell you a 'one size fits all' solution. Products include the RVG6000 digital $\mathrm{X}$-ray sensor from Kodak Dental Systems. It is designed to obtain extremely accurate images and take advantage of all the possibilities of digital radiography in terms of image processing, integration and data exchange.

Reader response number 55

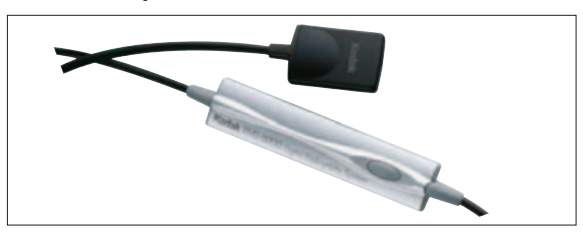

\section{Enhanced controls}

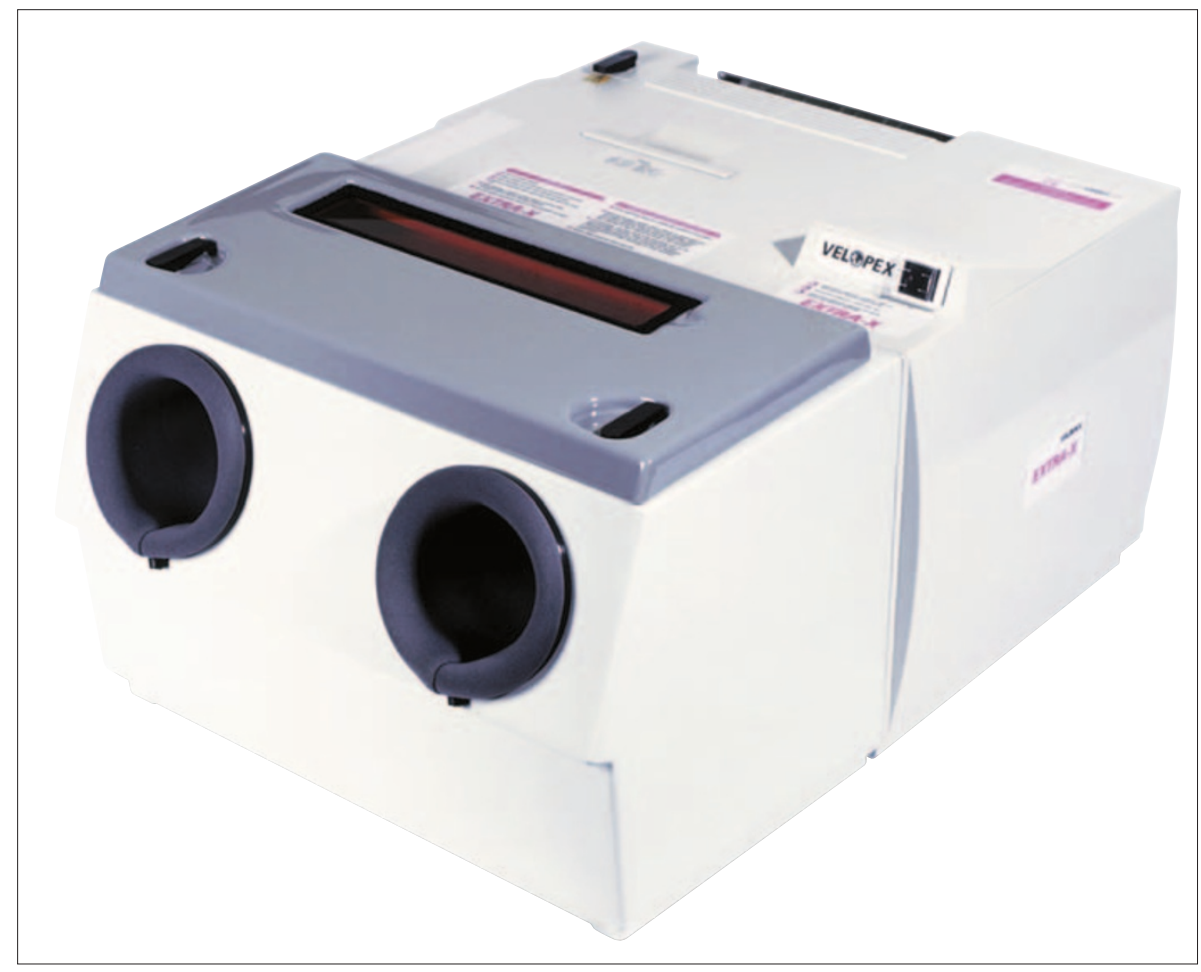

The Velopex Extra-X automatic daylight Xray film processor can process all sizes of dental film from intra-oral to panoramic. It has had a recent redesign of its control panel and decals to ensure ergonomic efficiency and at-a-glance clarity.

The machine's aesthetics have also received attention, and the Velopex

\section{Understanding treatment options}

Kodak Dental Systems range of products includes the Kodak 8000 digital panoramic system. The unit is controlled from the surgery computer, provides high quality images in real time and is simple to operate. The Kodak's DX6490 dental digital camera system is specifically designed for dental photography. The advanced camera system enables the capture, printing and sharing of sharp, 4.0megapixel images with the push of a button, while the Kodak 1000 intraoral video camera is a precision tool which enhances patient communication by capturing and displaying details invisible to the naked eye.

The digital imaging products integrate with Kodak practice management software, forming a combination which captures, displays, prints and shares images providing patients with a greater understanding of treatment options.

Reader response number 57

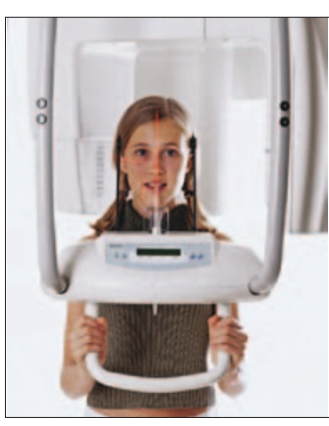




\section{Clearer patient communication}

The ClearVu Connect from DPS allows you to enhance your treatment plans, enabling complete communication with your patients as they will be able to understand treatment explanations far more easily by seeing their own mouth displayed on screen.

This lightweight, portable unit captures 112 high resolution images at the press of a button and will produce clear images even if your patient should move. The 1800 rotating tip allows you to capture images in both upper and lower areas without having to change your grip.

The product also provides an excellent depth of field and allows you to take intraoral or extraoral images. Due to the proprietary heat transference system the lens will not become foggy and the tip is fully autoclaveable so there is no need to use barrier sheaths.

\section{In-house installation}

The Orthophos XG Plus unit from Sirona Dental Systems features a novel patient positioning system allowing easy operation to give the best imaging results and incorporating a unique, electronic colour touch screen to simplify functions which helps to eliminate operator errors.

The user interface enables the operator to choose between standard visualisation, constant magnification and artefact-free images. The operator also has the facility to manually preselect the patient's jaw shape. In conjunction with Sidexis, the software compatible with all leading practice management systems, it enables the whole imaging and processing function to be contained and documented in one compact unit.

\section{Versatile mounts}

Dentsply Rinn provides a wide range of vinyl $\mathrm{x}$-ray film mounts, with a variety of configurations to suit your needs. Designs available include arrangements for anything from one to sixteen bitewings, an occlusal mount and a selection for full mouth series. Alternatively, Rinn's Universal Cut-Apart mounts will hold any configuration of mounts. In addition the Rinn Universal Collimator clips onto any roundheaded long-cone $\mathrm{x}$-ray unit, converting it from round to the recommended rectangular collimation. With rectangular collimation a beam alignment device, such as Dentsply's XCP kit, can be used to ensure the $\mathrm{X}$-ray film is properly aligned. Correct beam alignment reduces the chance of 'cone cutting' or distortion on the $\mathrm{x}$-ray image.

Reader response number 60

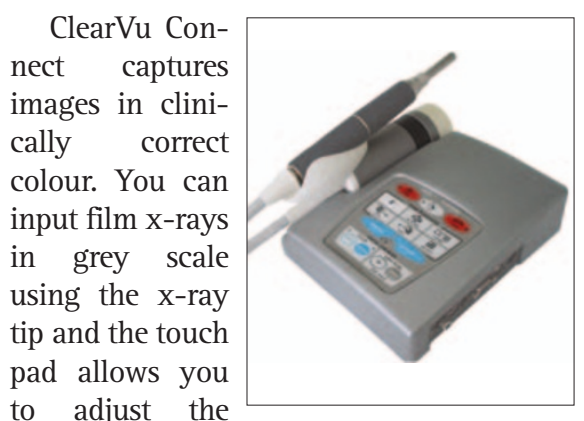
contrast quickly and easily. You can also reverse and invert picture images using the touch pad making them anatomically correct and easier for the patient to understand. It has three video outputs, VGA and USB ports and you can also download images onto a standard Smart Card.

Reader response number 58

By combining all the information into one snapshot image, the treatment can be explained in much clearer detail with greater acceptance and understanding by the patient.

Reader response number 59

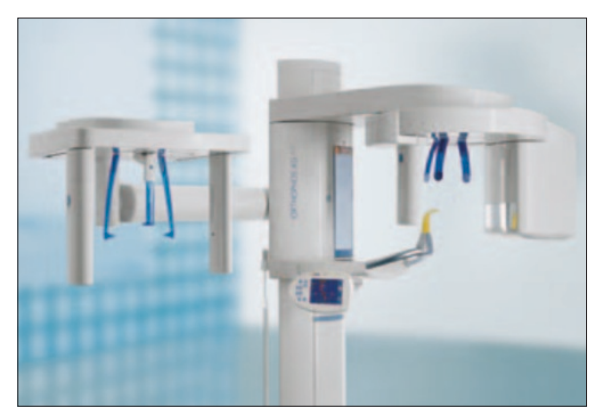

\section{Wireless imaging}

The Schick CDR Wireless is a digital $\mathrm{x}$ ray sensor which does not need a cable. Instead radio waves are used to transmit intra-oral images directly to the computer via a base station. No cable means greater chairside freedom for the dentist and the sensor is easier to use. It can also be used with the Schick CDR digital x-ray system and produces the same razorsharp images from its unique Active Pixel Sensor technology.

Reader response number $\mathbf{6 1}$

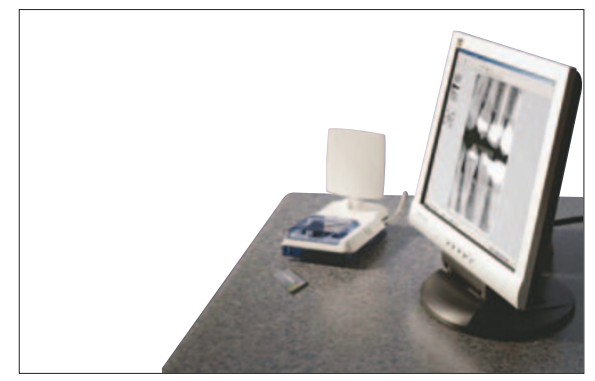

